\title{
Spectraloid operator polynomials, the approximate numerical range and an Eneström-Kakeya theorem in Hilbert space
}

by

\author{
Jan Swoboda (Bonn) and Harald K. Wimmer (Würzburg)
}

\begin{abstract}
We study a class of operator polynomials in Hilbert space which are spectraloid in the sense that spectral radius and numerical radius coincide. The focus is on the spectrum in the boundary of the numerical range. As an application, the EneströmKakeya-Hurwitz theorem on zeros of real polynomials is generalized to Hilbert space.
\end{abstract}

1. Introduction. For many purposes the Eneström-Kakeya theorem ([31, p. 137], [37, p. 4], [9, p. 12], [38, p. 255]) is an effective criterion to test whether a real polynomial has all its zeros in the unit disk. It can be stated as follows.

THEOREM 1.1. Let

$$
h(z)=\sum_{j=0}^{m-1} a_{j} z^{j}
$$

be a real polynomial such that

$$
0<a_{0} \leq a_{1} \leq \cdots \leq a_{m-1} .
$$

Then all zeros $\lambda$ of $h(z)$ satisfy $|\lambda| \leq 1$.

The theorem has numerous applications, which range from asymptotics of partial sums of power series [11] or a local-global stability principle for discrete-time systems [28] to coding theory [13], the economic theory of depreciation and reinvestment [41, stability analysis of delay filters [36] and models of high energy collisions [10] in physics. In this paper we are concerned with an extension of the Eneström-Kakeya theorem to operators in

2010 Mathematics Subject Classification: 47A10, 47A56, 47A12.

Key words and phrases: operator polynomials, Eneström-Kakeya theorem, normal approximate eigenvalues, semisimple eigenvalues, Jordan chains, approximate point spectrum, residual spectrum, spectral radius, numerical range, numerical radius, spectraloid operator. 
Hilbert space, which is different from the ones given by Furuta and Nakamura [21] and by Fujii and Kubo [18. Our starting point is a sharper version of Theorem 1.1, which goes back to Hurwitz [26] (see also [1], [2]). We use the following notation, which later will be extended to operator polynomials. For a complex polynomial $p(z)$ we define

$$
\sigma(p)=\{\lambda \in \mathbb{C} ; p(\lambda)=0\} \quad \text { and } \quad r(p)=\max \{|\lambda| ; \lambda \in \sigma(p)\} .
$$

Let $\pi_{m-1}^{+}$denote the set of all real polynomials $p(z)=\sum_{j=0}^{m-1} a_{j} z^{j}$ satisfying (1.2).

TheOREM $1.2([26])$. Let $h(z)=a_{0}+a_{1} z+\cdots+a_{m-1} z^{m-1}$ be a real polynomial with

$$
\begin{aligned}
0<a_{0}=a_{1}=\cdots=a_{r_{1}-1}<a_{r_{1}}= & a_{r_{1}+1}=\cdots=a_{r_{2}-1}<\cdots \\
& <a_{r_{s}}=a_{r_{s}+1}=\cdots=a_{m-1} .
\end{aligned}
$$

Then $r(h) \leq 1$. Set $k=\operatorname{gcd}\left(r_{1}, \ldots, r_{s}, m\right)$. Then $r(h)=1$ if and only if $k>1$. In that case

$$
0<a_{0}=\cdots=a_{k-1} \leq a_{k}=\cdots=a_{2 k} \leq \cdots \leq a_{m-k}=\cdots=a_{m-1},
$$

and

$$
h(z)=\left(1+z+\cdots+z^{k-1}\right) p\left(z^{k}\right), \quad p \in \pi_{\ell-1}^{+}, \ell=m / k,
$$

and $p(z)$ has no zeros $\lambda$ with $|\lambda|=1$. The zeros of $h(z)$ on the unit circle are simple, and they are the nontrivial $k$ th roots of unity, $e^{2 \nu \pi i / k}, \nu=$ $1, \ldots, k-1$.

To prove Theorem 1.2 one can assume that $a_{m-1}=1$, and then use a multiplier $z-1$ and consider the polynomial $g(z)=(z-1) h(z)$. Set $a_{-1}=0$. Then

$$
g(z)=z^{m}-\sum_{j=0}^{m-1}\left(a_{j}-a_{j-1}\right) z^{j},
$$

and $\sigma(g)=\sigma(h) \cup\{1\}$. Therefore 1.2 implies $g(z)=z^{m}-\sum_{j=0}^{m-1} c_{j} z^{j}$ with $c_{0}>0, c_{j} \geq 0, j=1, \ldots, m-1$, and

$$
\sum_{j=0}^{m-1} c_{j}=1 \text {. }
$$

Because of (1.4) it is more convenient to deal with $g(z)$ instead of the polynomial $h(z)$ in (1.1). Therefore in this paper the focus is on operator polynomials of the form

$$
G(z)=I z^{m}-\sum_{j=0}^{m-1} C_{j} z^{j},
$$


where the coefficients $C_{j}$ are bounded, positive semidefinite operators on a Hilbert space. We shall extend the following theorem to operator polynomials, and then generalize Theorem 1.2 to Hilbert spaces.

Theorem 1.3 ([26], [1], [35, p. 92]). Let

$$
g(z)=z^{m}-\left(c_{m-1} z^{m-1}+\cdots+c_{1} z+c_{0}\right)
$$

be a real polynomial, $g(z) \neq z^{m}$. Set $c_{-1}=0$. Let $t \in\{0, \ldots, m-1\}$ be such that $c_{t}>0$ and $c_{j}=0$ if $j<t$. Suppose

$$
c_{j} \geq 0, \quad j=0, \ldots, m-1, \quad \text { and } \quad \sum_{j=t}^{m-1} c_{j} \leq 1 .
$$

Then:

(i) $r(g) \leq 1$.

(ii) $r(g)=1$ if and only if $1 \in \sigma(g)$, i.e. $\sum_{j=0}^{m-1} c_{j}=1$.

(iii) The zeros of $g(z)$ on the unit circle (if any) are simple.

(iv) Suppose $r(g)=1$. Define

$d=\operatorname{gcd}\left(\left\{j ; c_{t+j} \neq 0, j=0, \ldots, m-t-1\right\} \cup\{m-t\}\right), \quad \ell=(m-t) / d$.

Then

$$
\{\lambda ; g(\lambda)=0,|\lambda|=1\}=\left\{e^{2 \nu \pi i / d} ; \nu=0, \ldots, d-1\right\},
$$

and

$$
g(z)=z^{t}\left[z^{\ell d}-\sum_{j=0}^{\ell-1} c_{j d} z^{j d}\right]=z^{t}\left(z^{d}-1\right) p\left(z^{d}\right)
$$

with $r\left(p\left(z^{d}\right)\right)<1$. In particular, if $c_{0}>0$ then the zeros of $g(z)$ are $m$ th roots of unity.

The content of the paper is as follows. In Section 2 we recall basic concepts of spectral theory of operators in Hilbert space such as residual spectrum and approximate point spectrum. We define analogous concepts for the set $\sigma(B)=\{\lambda \in \mathbb{C} ; 0 \in \sigma(B(\lambda))\}$ of characteristic values of operator polynomials

$$
B(z)=\sum_{j=0}^{m} B_{j} z^{j} \in \mathcal{L}(H)[z] .
$$

Moreover, we introduce approximate characteristic values of $B(z)$ and corresponding approximate Jordan chains. In Section 3 we investigate the approximate numerical range of operator polynomials. It will be shown that the residual spectrum on the boundary of the numerical range is empty if the coefficients of $B(z)$ in $(1.8)$ are selfadjoint. In Section 4 we deal with operator polynomials 1.5 assuming $C_{j}=C_{j}^{*}, C_{j} \geq 0$ (positive semidefinite), 
and $\sum_{j=0}^{m-1} C_{j} \leq I$. We shall prove that $\sigma(G)$ is contained in the closed unit disk. Special attention will be given to the characteristic values of $G(z)$ on the unit circle $\partial \mathbb{D}$. It will be shown that they lie on the boundary of the numerical range of $G(z)$. Hence it will follow from results of Section 3 that characteristic values on $\partial \mathbb{D}$ are in the normal approximate spectrum of $G(z)$, and that they are approximately semisimple. Moreover, $\partial \mathbb{D}$ does not contain residual characteristic values of $G(z)$. However, residual characteristic values may well exist in the interior of the unit disk, as we shall illustrate by an example. In Section 5 we extend the Eneström-Kakeya theorem to Hilbert space.

2. The spectrum, definitions and notation. Let $\mathbb{D}=\{z \in \mathbb{C}$; $|z|<1\}$ be the open unit disk and $\partial \mathbb{D}=\{z \in \mathbb{C} ;|z|=1\}$ the unit circle of the complex plane. The set of nonnegative real numbers will be denoted by $\mathbb{R}_{\geq}$. Let $E_{m}=\left\{\zeta \in \mathbb{C} ; \zeta^{m}=1\right\}$ be the group of $m$ th roots of unity. If $\zeta \in E_{m}$ then ord $\zeta$ will denote the order of $\zeta$.

Let $H$ be a complex Hilbert space and $S_{H}=\{x \in H ;\|x\|=1\}$ its unit sphere, and let $\mathcal{L}(H)$ be the algebra of bounded linear operators on $H$. If $v \in H$ then $v^{*} \in H^{*}$ is defined by $v^{*}(u)=\langle v, u\rangle$ for all $u \in H$. If $T \in \mathcal{L}(H)$ then $T^{*}$ denotes the adjoint of $T$. We say that an operator $T \in \mathcal{L}(H)$ is positive semidefinite $(T \geq 0)$ if $T$ is selfadjoint and satisfies $\langle x, T x\rangle \geq 0$ for all $x \in H$. If $\langle x, T x\rangle>0$ for all $x \in H, x \neq 0$, then we write $T>0$. A selfadjoint operator $T$ will be called strictly positive definite $(T \gg 0)$ if

$$
\langle x, T x\rangle \geq \delta\langle x, x\rangle \quad \text { for some } \delta>0 .
$$

It follows from [5, p. 244, (57.16)] that $T \gg 0$ if and only if $T>0$ and $T$ is invertible. If $T \geq 0$ then (see [40, p. 314], [43, p. 63]) there exists a unique positive semidefinite square root, and if $T$ is strictly positive definite then $T^{1 / 2} \gg 0$. Let $S, T \in \mathcal{L}(H)$ be selfadjoint. We write $S \geq T$ if $S-T \geq 0$ and $S \gg T$ if $S-T \gg 0$.

Let $\sigma(T)$ be the spectrum of $T \in \mathcal{L}(H)$ and let

$$
\sigma_{P}(T)=\{\lambda \in \mathbb{C} ; \lambda I-T \text { is not injective }\}
$$

be the point spectrum of $T$. A complex number $\lambda$ is called an approximate eigenvalue of $T$ if for all $\epsilon>0$ there exists a $u \in H$ such that

$$
\|(\lambda I-T) u\|<\epsilon\|u\| .
$$

The set $\sigma_{A}(T)$ of approximate eigenvalues of $T$ is the approximate point spectrum of $T$ (see [6], [24, p. 54], [5, p. 241], [34, p. 413], [20, p. 81]). We say that a sequence $v=\left(v_{\nu}\right) \in H^{\mathbb{N}}$ is an approximate eigenvector corresponding to $\lambda$ if

$$
\lim _{\nu \rightarrow \infty}(\lambda I-T) v_{\nu}=0 \quad \text { and } \quad v \neq 0 \text { (null sequence). }
$$


If convenient, one can assume $\left\|v_{\nu}\right\|=1, \nu \in \mathbb{N}$. Evidently, $\sigma_{P}(T) \subseteq \sigma_{A}(T)$. Let

$$
\sigma_{R}(T)=\{\lambda \in \mathbb{C} ; \lambda I-T \text { is injective and } \overline{\operatorname{range}(\lambda I-T)} \neq H\}
$$

be the residual spectrum of $T$. Then (see e.g. [34, p. 413])

$$
\sigma(T)=\sigma_{A}(T) \cup \sigma_{R}(T) .
$$

It is known ([39, p. 194], [15, p. 161]) that

$$
\sigma_{R}(T) \subseteq \sigma_{P}\left(T^{*}\right)
$$

The following notation will be useful. Let $u=\left(u_{\nu}\right), v=\left(v_{\nu}\right) \in H^{\mathbb{N}}$. We write

$$
u \hat{=} v \quad \text { if } \quad \lim _{\nu \rightarrow \infty}\left(u_{\nu}-v_{\nu}\right)=0 .
$$

Then $u \hat{=} 0$ denotes a null sequence. Let

$$
H[z]=\left\{f: \mathbb{C} \rightarrow H ; f(z)=\sum_{i=0}^{k} f_{i} z^{i}, f_{i} \in H, k \in \mathbb{N}_{0}\right\} .
$$

If $p(z)=\left(p_{\nu}(z)\right), q(z)=\left(q_{\nu}(z)\right) \in(H[z])^{\mathbb{N}}$ then we write

$$
p(z) \hat{=} q(z) \quad \text { if } \quad \lim _{\nu \rightarrow \infty} p_{\nu}(z)=\lim _{\nu \rightarrow \infty} q_{\nu}(z) \text { for all } z \in \mathbb{C} .
$$

According to $(2.1)$ we have $\lambda \in \sigma_{A}(T)$ if and only if $(\lambda I-T) v \hat{=} 0$ for some $v \hat{\neq} 0$. We define

$$
\operatorname{Ker}_{A}(\lambda I-T)=\left\{v \in H^{\mathbb{N}} ;(\lambda I-T) v \hat{=} 0\right\},
$$

and we write $\operatorname{Ker}_{A}(\lambda I-T) \hat{=}\{0\}$ if $\lambda \notin \sigma_{A}(T)$.

Let $H$ be finite-dimensional, say $H=\mathbb{C}^{n}$, and let $T \in \mathbb{C}^{n \times n}$. The ascent of an eigenvalue $\lambda$ of $T$ is the smallest integer $\ell$ such that $\operatorname{Ker}(\lambda I-T)^{\ell+1}=$ $\operatorname{Ker}(\lambda I-T)^{\ell}$. An eigenvalue $\lambda$ with ascent 1 is called semisimple [12]. In that case we have

$$
\operatorname{Ker}(\lambda I-T)^{2}=\operatorname{Ker}(\lambda I-T),
$$

and the space $\mathbb{C}^{n}$ splits into $T$-invariant subspaces $V$ and $W$ such that

$$
\mathbb{C}^{n}=V \oplus W \quad \text { and } \quad T_{\mid V}=\lambda I, \quad \lambda \notin \sigma\left(T_{\mid W}\right) .
$$

If $H$ is an arbitrary Hilbert space, we say that $\lambda \in \sigma_{A}(T)$ is approximately semisimple if

$$
\operatorname{Ker}_{A}(\lambda I-T)^{2}=\operatorname{Ker}_{A}(\lambda I-T) .
$$

It is easy to see that the identity $(2.5)$ can be described in terms of pairs $(v, w) \in H^{\mathbb{N}} \times H^{\mathbb{N}}$ satisfying (2.7) below. 
Lemma 2.1. Let $\lambda \in \sigma_{A}(T)$.

(i) We have (2.5) if and only if

$$
\operatorname{Ker}_{A}\left(\begin{array}{cc}
\lambda I-T & 0 \\
I & \lambda I-T
\end{array}\right)=\left\{\left(\begin{array}{c}
v \\
w
\end{array}\right) ; v \hat{=} 0,(\lambda I-T) w \hat{=} 0\right\} .
$$

(ii) Conversely, $\operatorname{Ker}_{A}(\lambda I-T) \subsetneq \operatorname{Ker}_{A}(\lambda I-T)^{2}$ if and only if there exist sequences $v, w \in H^{\mathbb{N}}$ such that

$$
(\lambda I-T) v \hat{=} 0, \quad v \hat{\neq} 0, \quad(\lambda I-T) w \hat{=} v .
$$

We call the pair $(v, w)$ in (2.7) an approximate Jordan chain of length 2. Note that the sequence $v$ is an approximate eigenvector. Hence $\lambda$ is approximately semisimple if and only if the corresponding approximate eigenvectors cannot be extended to approximate Jordan chains of length 2 .

If there exists a sequence $v \in H^{\mathbb{N}}$ such that $v \hat{\neq} 0$, and $(\lambda I-T) v \hat{=} 0$ and $(\lambda I-T)^{*} v \hat{=} 0$, then $\lambda$ is called a normal approximate eigenvalue of $T$ (see e.g. [16], [19], 30]). The case where $(\lambda I-T) v \hat{=} 0$ is equivalent to $(\lambda I-T)^{*} v \hat{=} 0$ is of special interest. First consider $H=\mathbb{C}^{n}$ and $T \in \mathbb{C}^{n \times n}$. Then

$$
\operatorname{Ker}(\lambda I-T)^{*}=\operatorname{Ker}(\lambda I-T)
$$

is equivalent to

$$
[\operatorname{range}(\lambda I-T)]^{\perp}=\operatorname{Ker}(\lambda I-T) .
$$

Hence (2.8) holds if and only if

$$
\mathbb{C}^{n}=\operatorname{Ker}(\lambda I-T)(1) \text { range }(\lambda I-T),
$$

or equivalently, if and only if there exists a unitary operator $U$ such that

$$
U^{*} T U=\left(\begin{array}{cc}
\lambda I & 0 \\
0 & T_{2}
\end{array}\right), \quad \lambda \notin \sigma\left(T_{2}\right) .
$$

Lemma 2.2. Let $\lambda \in \sigma_{A}(T)$. If

$$
\operatorname{Ker}_{A}(\lambda I-T)=\operatorname{Ker}_{A}(\lambda I-T)^{*}
$$

then $\lambda$ is approximately semisimple.

Proof. Suppose $w \in \operatorname{Ker}_{A}(\lambda I-T)^{2}$. Then

$$
0 \hat{=}(\lambda I-T)[(\lambda I-T) w]=(\lambda I-T)^{*}(\lambda I-T) w .
$$

Therefore $\|(\lambda I-T) w\|^{2} \hat{=} 0$. Hence $(\lambda I-T) w \hat{=} 0$, and we have 2.5).

Let $\mathcal{L}(H)[z]$ be defined in accordance with 2.4 . Then $B(z) \in \mathcal{L}(H)[z]$ is an operator polynomial of degree $m$ if

$$
B(z)=\sum_{j=0}^{m} B_{j} z^{j},
$$


and $B_{0}, \ldots, B_{m} \in \mathcal{L}(H), B_{m} \neq 0$. Set $B^{*}(z)=\sum_{j=0}^{m} B_{j}^{*} z^{j}$. We extend the notion of spectrum from operators $T \in \mathcal{L}(H)$ to operator polynomials 2.10 with invertible leading coefficient $B_{m}$. We define

$$
\sigma(B)=\{\lambda \in \mathbb{C} ; B(\lambda) \text { is not invertible }\}=\{\lambda \in \mathbb{C} ; 0 \in \sigma(B(\lambda))\},
$$

and $r(B)=\sup \{|\lambda| ; \lambda \in \sigma(B)\}$, and

$$
\sigma_{M}(B)=\left\{\lambda ; 0 \in \sigma_{M}(B(\lambda))\right\} \quad \text { for } M \in\{P, A, R\} .
$$

Thus $\lambda \in \sigma_{A}(B)$ if and only if

$$
\sum_{j=0}^{m} B_{j} \lambda^{j} v \hat{=} 0
$$

for some sequence $v \in H^{\mathbb{N}}, v \hat{\neq} 0$. Adapting a notion of [4] we call the elements of $\sigma_{A}(B)$ approximate characteristic values of $B(z)$. If (2.11) holds then we say that $v$ is an approximate eigenvector of $B(z)$ corresponding to $\lambda$. For operator polynomials we define (approximate) semisimplicity in terms of Jordan chains. Let $\lambda \in \sigma_{A}(B)$. If $v, w \in H^{\mathbb{N}}$ are sequences such that

$$
B(\lambda) v \hat{=} 0, \quad v \hat{\neq} 0, \quad B^{\prime}(\lambda) v+B(\lambda) w \hat{=} 0,
$$

then $(v, w)$ is called an approximate Jordan chain of length 2 of $B(z)$ corresponding to $\lambda$. Thus, all approximate Jordan chains of $\lambda$ have length 1 if and only if

$$
\operatorname{Ker}_{A}\left(\begin{array}{cc}
B(\lambda) & 0 \\
B^{\prime}(\lambda) & B(\lambda)
\end{array}\right)=\left\{\left(\begin{array}{c}
v \\
w
\end{array}\right) ; v \hat{=} 0, B(\lambda) w \hat{=} 0\right\} .
$$

(We refer to [4] or [27] for a study of Jordan chains of operator polynomials.) If $B(z)=\lambda I-T$ then 2.12 reduces to $(2.6)$. We say that $\lambda$ is approximately semisimple if there are no corresponding approximate Jordan chains of length 2.

In our examples we shall deal with $\ell_{2}=\ell_{2}(\mathbb{C})$, the complex Hilbert space of square summable sequences. Let $e_{1}=(1,0,0, \ldots)^{T}, e_{2}=(0,1,0, \ldots)^{T}$, etc., be the standard orthonormal basis of $\ell_{2}$. Define $e=\left(e_{\nu}\right)_{\nu \in \mathbb{N}}$.

Example 2.3. Consider $H=\ell_{2}$ and $G(z)=z^{3} I-\left(C_{2} z^{2}+C_{1} z+C_{0}\right)$ with

$C_{0}=C_{1}=\operatorname{diag}(1 / 2,1 / 3,1 / 4, \ldots), \quad C_{2}=I-2 C_{0}=\operatorname{diag}(0,1 / 3,2 / 4, \ldots)$.

Then $G(0)=G^{\prime}(0)=C_{0}$ implies

$$
\lim _{\nu \rightarrow \infty} G(0) e_{\nu}=0 \quad \text { and } \quad \lim _{\nu \rightarrow \infty}\left(G^{\prime}(0) e_{\nu}+G(0) e_{\nu}\right)=0 .
$$

Hence $(e, e)$ is an approximate Jordan chain of length 2 corresponding to $0 \in \sigma(G)$. 
Let $\lambda \in \sigma_{A}(B)$, and suppose $B(\lambda) v \hat{=} 0$ and $B(\lambda)^{*} v \hat{=} 0$ for some $v \hat{\neq} 0$. Then the approximate characteristic value $\lambda$ will be called normal, and $v$ is a corresponding normal approximate eigenvector. To illustrate the preceding concepts we consider a monic operator polynomial of degree 2 .

EXAmple 2.4. Let $H=\ell_{2}$ and consider $G(z)=z^{2} I-\left(C_{1} z+C_{0}\right)$ with $C_{1}=\operatorname{diag}(1 / 2,1 / 3,1 / 4,1 / 5, \ldots) \quad$ and $\quad C_{0}=\operatorname{diag}(0,1 / 3,2 / 4,3 / 5, \ldots)$. Then $C_{1} \geq 0, C_{0} \geq 0$, and $C_{0}=I-2 C_{1}$. Moreover, $C_{0}+C_{1}=I-C_{1} \leq I$. From $G(1)=C_{1}$ and $G(-1)=3 C_{1}$ it follows that $e$ is a normal approximate eigenvector of $G(z)$ corresponding to 1 and to -1 . But $\pm 1 \notin \sigma_{P}(G)$. Set $p_{\nu}(z)=[(z-1)-1 / \nu] e_{\nu}$ and $p(z)=\left(p_{\nu}(z)\right)$. Then $G(z) e \hat{=}(z+1) p(z)$.

The following proposition extends Lemma 2.2 .

Proposition 2.5. Let $\lambda \in \sigma_{A}(B)$ be such that

$$
\operatorname{Ker}_{A} B(\lambda)=\operatorname{Ker}_{A} B(\lambda)^{*} \text {. }
$$

Then $\lambda$ is approximately semisimple if

$$
\lim _{\nu \rightarrow \infty} v_{\nu}^{*} B^{\prime}(\lambda) v_{\nu} \neq 0
$$

for all $v=\left(v_{\nu}\right) \in \operatorname{Ker}_{A} B(\lambda), v \hat{\neq} 0$.

Proof. Suppose $v, w \in H^{\mathbb{N}}$ and

$$
B(\lambda) v \hat{=} 0, \quad v \hat{\neq} 0, \quad B^{\prime}(\lambda) v+B(\lambda) w \hat{=} 0 .
$$

Then $\lim _{\nu \rightarrow \infty} v_{\nu}^{*} B^{\prime}(\lambda) v_{\nu}=0$, in contradiction to 2.14.

If $B(z)=z I-T$ and $T \in \mathcal{L}(H)$, then $B^{\prime}(z)=I$. This implies (2.14) for all $\lambda \in \mathbb{C}$, and we recover Lemma 2.2 .

3. The approximate numerical range. For an operator polynomial $B(z) \in \mathcal{L}(H)[z]$ we define the approximate numerical range $W_{A}(B)$ and the numerical range $W(B)$ as

$$
W_{A}(B)=\left\{\lambda \in \mathbb{C} ; \lim _{\nu \rightarrow \infty} y_{\nu}^{*} B(\lambda) y_{\nu}=0 \text { for some } y=\left(y_{\nu}\right) \in H^{\mathbb{N}}, y \hat{\neq} 0\right\}
$$

and

$$
W(B)=\left\{\lambda \in \mathbb{C} ; y^{*} B(\lambda) y=0 \text { for some } y \in H, y \neq 0\right\} .
$$

For polynomial matrices $B(z) \in \mathbb{C}^{n \times n}[z]$ the concept of numerical range was first studied systematically in [29] and investigated further in [32], [17], [33]. If $B(z)=z I-T$ and $T \in \mathcal{L}(H)$, then $W_{A}(B)$ and $W(B)$ are equal to

$$
F_{A}(T)=\left\{\lambda \in \mathbb{C} ; \lambda=\lim x_{\nu}^{*} T x_{\nu} \text { for some }\left(x_{\nu}\right) \in H^{\mathbb{N}},\left\|x_{\nu}\right\|=1, \nu \in \mathbb{N}\right\}
$$

and

$$
\begin{aligned}
F(T) & =\left\{\lambda \in \mathbb{C} ; \lambda=x^{*} T x \text { for some } x \in H,\|x\|=1\right\} \\
& =\left\{x^{*} T x ; x \in H,\|x\|=1\right\}
\end{aligned}
$$


respectively. The set $F(T)$ is known as the numerical range (or field of values) of $T$. By the Toeplitz-Hausdorff theorem $F(T)$ is convex (see e.g. [22, p. 4], [3, p. 388]). According to [29] the set $W(B)$ is bounded if and only if $0 \notin F\left(B_{m}\right)$. Let

$$
w(B)=\sup \{|\lambda| ; \lambda \in W(B)\}
$$

be the numerical radius of $B(z)$. Evidently,

$$
\sigma_{A}(B) \subseteq W_{A}(B) .
$$

The next example shows that, in general, $W(B)$ is a proper subset of $W_{A}(B)$.

Example 3.1. Consider $H=\ell_{2}$, and

$$
T=\operatorname{diag}(1 / 2,2 / 3,3 / 4, \ldots)
$$

and $B(z)=z I-T$. Let $e=\left(e_{\nu}\right)$. Then $e \in\left(S_{H}\right)^{\mathbb{N}}$ and $B(1) e \hat{=} 0$. Hence $1 \in \sigma_{A}(B)$, and therefore $1 \in W_{A}(B)$. Let $u=\left(u_{1}, u_{2}, \ldots,\right)^{T} \in \ell_{2}$. Then $\sum_{k=1}^{\infty}\left|u_{k}\right|^{2}=1$ implies $u^{*} T u<1$. Hence $1 \notin W(B)$.

The following theorem provides an intrinsic characterization of $\overline{W(B)}$. We point out a general result of [7] on the closure of the numerical range of operators in Banach spaces and we also refer to corresponding comments in [5, p. 329].

Proposition 3.2. We have $W_{A}(B)=\overline{W(B)}$.

Proof. Let us first show that $W_{A}(B) \subseteq \overline{W(B)}$. Suppose $\lambda \in W_{A}(B)$ and let $\left(v_{\nu}\right) \in\left(S_{H}\right)^{\mathbb{N}}$ be a corresponding sequence with $\lim _{\nu \rightarrow \infty} v_{\nu}^{*} B(\lambda) v_{\nu}=0$. The sequences $\left(v_{\nu}^{*} B_{j} v_{\nu}\right), j=0, \ldots, m$, are bounded. We can assume that the limits $\beta_{j}=\lim _{\nu \rightarrow \infty} v_{\nu}^{*} B_{j} v_{\nu}, j=0, \ldots, m$, exist. Hence

$$
\beta_{j}=v_{\nu}^{*} B_{j} v_{\nu}+\epsilon_{j \nu} \quad \text { and } \quad \lim _{\nu \rightarrow \infty} \epsilon_{j \nu}=0 .
$$

Define

$$
b_{\nu}(z)=\sum_{j=0}^{m} v_{\nu}^{*} B_{j} v_{\nu} z^{j}, \quad \nu \in \mathbb{N}, \quad \text { and } \quad b(z)=\sum_{j=0}^{m} \beta_{j} z^{j} .
$$

Then $b_{\nu}(z)=\sum_{j}\left(\beta_{j}-\epsilon_{j \nu}\right) z^{j}$, and $b(\lambda)=0$. Zeros of a complex polynomial vary continuously with its coefficients (see e.g. [8, p. 230]). Hence, there exists a sequence $\left(\lambda_{\nu}\right)$ such that

$$
b_{\nu}\left(\lambda_{\nu}\right)=0, \quad\left|\lambda-\lambda_{\nu}\right|<\delta_{\nu}, \quad \text { and } \quad \lim _{\nu \rightarrow \infty} \delta_{\nu}=0 .
$$

Because of $b_{\nu}\left(\lambda_{\nu}\right)=v_{\nu}^{*} B\left(\lambda_{\nu}\right) v_{\nu}$ we have $\lambda_{\nu} \in W(B)$. Therefore $\lambda=$ $\lim _{\nu \rightarrow \infty} \lambda_{\nu} \in \overline{W(B)}$.

We turn to the inclusion $\overline{W(B)} \subseteq W_{A}(B)$. Let $\lambda \in \overline{W(B)}$ and $\lambda_{\nu} \in$ $W(B), \nu \in \mathbb{N}$, be such that $\lim _{\nu \rightarrow \infty} \lambda_{\nu}=\lambda$. For each $\nu$ we have a $v_{\nu} \in S_{H}$ 
such that $v_{\nu}^{*} B\left(\lambda_{\nu}\right) v_{\nu}=0$. Hence

$$
\begin{aligned}
\left|v_{\nu}^{*} B(\lambda) v_{\nu}\right| & \leq\left|v_{\nu}^{*}\left(B(\lambda)-B\left(\lambda_{\nu}\right)\right) v_{\nu}\right|+\left|v_{\nu}^{*} B\left(\lambda_{\nu}\right) v_{\nu}\right| \\
& \leq\left\|B(\lambda)-B\left(\lambda_{\nu}\right)\right\|+\left|v_{\nu}^{*} B\left(\lambda_{\nu}\right) v_{\nu}\right| .
\end{aligned}
$$

We conclude that $\lim _{\nu \rightarrow \infty} v_{\nu}^{*} B(\lambda) v_{\nu}=0$, that is, $\lambda \in W_{A}(B)$.

From Proposition 3.2 we get $\partial W_{A}(B)=\partial W(B)$ and $\partial F_{A}(T)=\partial F(T)$. Moreover,

$$
w(B)=\sup \left\{|\lambda| ; \lambda \in W_{A}(B)\right\},
$$

and if $W(B)$ is bounded then

$$
w(B)=\max \left\{|\lambda| ; \lambda \in W_{A}(B)\right\} .
$$

It is known (see e.g. [20, p. 97]) that the spectrum of $T$ is contained in the closure of $F(T)$. A corresponding result holds for operator polynomials.

Lemma 3.3. We have $\sigma(B) \subseteq W_{A}(B)$ and $r(B) \leq w(B)$.

Proof. From (2.2) we obtain $\sigma(B)=\sigma_{A}(B) \cup \sigma_{R}(B)$. Therefore, by (3.1), it suffices to prove $\sigma_{R}(B) \subseteq W_{A}(B)$. Suppose $\lambda \in \sigma_{R}(B)$, that is, $0 \in$ $\sigma_{R}(B(\lambda))$. Then (2.3) implies $0 \in \sigma_{P}\left(B(\lambda)^{*}\right)$, that is, $\bar{\lambda} \in \sigma_{P}\left(B^{*}\right)$. Hence $\bar{\lambda} \in W_{A}\left(B^{*}\right)$. This is equivalent to $\lambda \in W_{A}(B)$.

We say that the operator polynomial $B(z)$ is spectraloid if

$$
w(B)=r(B) .
$$

If $B(z)=z I-T, T \in \mathcal{L}(H)$, then (3.4) is equivalent to $w(T)=r(T)$, and the operator $T$ is spectraloid in the sense of [23, p. 176], [22, p. 150], [20, p. 99].

In the following we are concerned with approximate characteristic values of $B(z)$ lying on the boundary of $W(B)$. We need an extension of Theorem 1.1 of [32], which will be proved along the lines of [32]. If $z_{i} \in \mathbb{C}$, $i=1,2,3$, then $\left[z_{1}, z_{2}, z_{3}\right]$ shall denote the triangle with vertices $z_{1}, z_{2}, z_{3}$. The interior of a set $M$ will be denoted by int $M$.

Lemma 3.4. If $\lambda \in \partial W(B)$ then $0 \in \partial F(B(\lambda))$.

Proof. Let us show first that 0 is not an interior point of $F_{A}(B(\lambda))$. Suppose there exists a disk $U(0, \epsilon)=\{w \in \mathbb{C} ;|w|<\epsilon\}$ such that $U(0, \epsilon) \subseteq$ $F_{A}(B(\lambda))$. Then there exist $z_{i} \in U(0, \epsilon), i=1,2,3$, such that 0 is an interior point of the triangle $\left[z_{1}, z_{2}, z_{3}\right]$. We have

$$
z_{i}=\lim _{\nu \rightarrow \infty} x_{i \nu}^{*} B(\lambda) x_{i \nu}
$$

for some sequence $\left(x_{i \nu}\right) \in\left(S_{H}\right)^{\mathbb{N}}, i=1,2,3$. Set $z_{i \nu}=x_{i \nu}^{*} B(\lambda) x_{i \nu}, \nu \in \mathbb{N}$, $i=1,2,3$. Then 0 is in the interior of the triangle

$$
\left[z_{1 \nu}, z_{2 \nu}, z_{3 \nu}\right] \subseteq U(0, \epsilon)
$$


if $\nu$ is sufficiently large, $\nu \geq \nu_{0}$. By assumption, $\lambda$ is a boundary point of $W(B)$. Therefore there exists a sequence $\left(\lambda_{k}\right)_{k \in \mathbb{N}}$ such that $\lim _{k \rightarrow \infty} \lambda_{k}=\lambda$ and

$$
\lambda_{k} \notin W(B), \quad k \in \mathbb{N} .
$$

Then

$$
\lim _{k \rightarrow \infty} x_{i \nu}^{*} B\left(\lambda_{k}\right) x_{i \nu}=x_{i \nu}^{*} B(\lambda) x_{i \nu}=z_{i \nu}, \quad i=1,2,3 .
$$

Hence, if $\nu \geq \nu_{0}$ and $k$ is sufficiently large, $k \geq k_{0}$, then 0 is in the interior of

$$
\left[x_{1 \nu}^{*} B\left(\lambda_{k}\right) x_{1 \nu}, x_{2 \nu}^{*} B\left(\lambda_{k}\right) x_{2 \nu}, x_{3 \nu}^{*} B\left(\lambda_{k}\right) x_{3 \nu}\right] .
$$

We have

$$
x_{i \nu}^{*} B\left(\lambda_{k}\right) x_{i \nu} \in F\left(B\left(\lambda_{k}\right)\right), \quad i=1,2,3 .
$$

Since $F\left(B\left(\lambda_{k}\right)\right)$ is convex it follows that $0 \in F\left(B\left(\lambda_{k}\right)\right)$ for $k \geq k_{0}$. Hence $x^{*} B\left(\lambda_{k}\right) x=0$ for some $x \neq 0$, that is, $\lambda_{k} \in W(B)$, in contradiction to (3.5).

From $\lambda \in \partial W(B)$ it follows that $\lambda \in \overline{W(B)}=W_{A}(B)$. Hence $0 \in$ $F_{A}(B(\lambda))$. Then $0 \notin \operatorname{int} F_{A}(B(\lambda))$ implies $0 \in \partial F_{A}(B(\lambda))=\partial F(B(\lambda))$.

In the case where $H$ is finite-dimensional the following proposition can be found in [25, p. 235].

Proposition 3.5. If $\lambda \in \partial F(T) \cap \sigma_{A}(T)$ then

$$
\begin{aligned}
\operatorname{Ker}_{A}(\lambda I-T) & =\operatorname{Ker}_{A}(\lambda I-T)^{*}, \\
\operatorname{Ker}(\lambda I-T) & =\operatorname{Ker}(\lambda I-T)^{*} .
\end{aligned}
$$

Proof. If $(\lambda I-T) y=0, y \neq 0$, then $v=\left(v_{\nu}\right), v_{\nu}=y, \nu \in \mathbb{N}$, is an approximate eigenvector of $T$. Hence it suffices to prove (3.6), and to consider $\lambda=0$. Suppose 3.6 does not hold. Then there exists a sequence $x=\left(x_{\nu}\right)$ with $x_{\nu}^{*} x_{\nu}=1, \nu \in \mathbb{N}$, such that $T x \hat{=} 0$ and $y=T^{*} x \hat{\neq} 0$. If $y=\left(y_{\nu}\right)$ then $\lim _{\nu \rightarrow \infty} y_{\nu}^{*} x_{\nu}=0$. Since the sequences $\left(y_{\nu}^{*} y_{\nu}\right),\left(y_{\nu}^{*} T y_{\nu}\right)$ and $\left(x_{\nu}^{*} T y_{\nu}\right)$ are bounded we can assume right away that they are convergent. Set $v_{\nu}=\lambda x_{\nu}+\mu y_{\nu}$. Then

$$
v_{\nu}^{*} v_{\nu}=|\lambda|^{2}+|\mu|^{2} y_{\nu}^{*} y_{\nu}+\left(\bar{\lambda} \mu x_{\nu}^{*} y_{\nu}+\bar{\mu} \lambda y_{\nu}^{*} x_{\nu}\right)
$$

and

$$
\lim _{\nu \rightarrow \infty} v_{\nu}^{*} T v_{\nu}=\lim _{\nu \rightarrow \infty}\left(\bar{\lambda} \mu x_{\nu}^{*} T y_{\nu}+\bar{\mu} \mu y_{\nu}^{*} T y_{\nu}\right)=\lim _{\nu \rightarrow \infty}\left(\bar{\lambda} \mu y_{\nu}^{*} y_{\nu}+\bar{\mu} \mu y_{\nu}^{*} T y_{\nu}\right) .
$$

Set $c=\lim _{\nu \rightarrow \infty}\left\|y_{\nu}\right\|$. Then $c>0$. Let $\lim _{\nu \rightarrow \infty} y_{\nu}^{*} T y_{\nu}=\tau c^{2}$. Then

$$
\lim _{\nu \rightarrow \infty} v_{\nu}^{*} T v_{\nu}=c^{2}(\bar{\lambda} \mu+\bar{\mu} \mu \tau) \text {. }
$$

Define

$$
G=\left\{v=\lambda x+\mu y ; \lambda, \mu \in \mathbb{C}, v=\left(v_{\nu}\right),\left\|v_{\nu}\right\|=1, \nu \in \mathbb{N}\right\} .
$$


Then

$$
V=\left\{\lim _{\nu \rightarrow \infty} v_{\nu}^{*} T v_{\nu} ; v \in G\right\} \subseteq F_{A}(T) .
$$

From (3.8) and (3.9) we obtain

$$
V=\left\{c^{2}(\bar{\lambda} \mu+\bar{\mu} \mu \tau) ;|\lambda|^{2}+c^{2}|\mu|^{2}=1\right\} .
$$

Thus $u \in V$ if and only if

$$
u=(\lambda, c \mu)^{*}\left(\begin{array}{ll}
0 & c \\
0 & \tau
\end{array}\right)\left(\begin{array}{c}
\lambda \\
c \mu
\end{array}\right)
$$

and $|\lambda|^{2}+|c \mu|^{2}=1$. Set $M=\left(\begin{array}{ll}0 & c \\ 0 & \tau\end{array}\right)$. Then $V=F(M)$. If $\tau=0$ then (see [22, Chapter 1.1]) the set $F(M)$ is a disk with center 0 and radius $c / 2$. If $\tau \neq 0$ then $F(M)$ is an ellipse with foci at 0 and $\tau$ and minor axis $c$. Therefore 0 is an interior point of $F_{A}(T)$. Hence $0 \notin \partial F_{A}(T)$. Because of $\partial F_{A}(T)=\partial F(T)$ this is a contradiction.

We now assume $B(z)=B^{*}(z)$ such that

$$
B_{j}^{*}=B_{j}, \quad j=0, \ldots, m,
$$

in (2.11).

Lemma 3.6. If $B(z)=B^{*}(z)$ then each of the sets $W(B)$ and $W_{A}(B)$ is symmetric with respect to the real axis.

Proof. Because of $W_{A}(B)=\overline{W(B)}$ we only have to show that $\lambda \in W(B)$ implies $\bar{\lambda} \in W(B)$. Let $x \in H, x \neq 0$, be such that $x^{*} B(\lambda) x=0$. Thus $\lambda \in W(B)$. Define $b(z)=\sum_{j=0}^{m} x^{*} B_{j} x z^{j}$. Then 3.10 implies $b(z) \in \mathbb{R}[z]$. Hence $b(\lambda)=0$ yields $b(\bar{\lambda})=0$, that is, $\bar{\lambda} \in W(B)$.

Theorem 3.7. Assume $B(z)=B^{*}(z)$.

(i) Let $\lambda \in \partial W(B)$. Then $\lambda \notin \sigma_{R}(B)$, i.e.

$$
\sigma_{R}(B) \cap \partial W(B)=\emptyset .
$$

(ii) If $\lambda \in \partial W(B) \cap \sigma(B)$ then

$$
\operatorname{Ker}_{A} B(\lambda)=\operatorname{Ker}_{A} B(\lambda)^{*} \text { and } \operatorname{Ker} B(\lambda)=\operatorname{Ker} B(\lambda)^{*} \text {. }
$$

Proof. (i) Suppose there exists an element $\lambda \in \sigma_{R}(B) \cap \partial W(B)$. Then $0 \in \sigma_{R}(B(\lambda))$, and $\lambda \in \partial W\left(B^{*}\right)$. Hence $(2.3)$ implies $0 \in \sigma_{P}\left(B(\lambda)^{*}\right)$ and Lemma 3.4 implies $0 \in \partial F\left(B(\lambda)^{*}\right)$. Then (3.7) in Proposition 3.5 yields $0 \in \sigma_{P}(B(\lambda))$. This is a contradiction, since the sets $\sigma_{P}(B)$ and $\sigma_{R}(B)$ are disjoint. Therefore we have (3.11).

(ii) If $\lambda \in \sigma(B)$ lies on the boundary of $W(B)$ then (3.11) and (2.2) imply $\lambda \in \sigma_{A}(B)$, i.e. $0 \in \sigma_{A}(B(\lambda))$. Thus (3.12) follows from Proposition 3.5. 


\section{Semidefinite coefficients. Let}

$$
G(z)=I z^{m}-\left(C_{m-1} z^{m-1}+\cdots+C_{1} z+C_{0}\right)
$$

be a monic operator polynomial with selfadjoint positive semidefinite coefficients $C_{j} \in \mathcal{L}(H), j=0, \ldots, m-1$.

4.1. The numerical radius. We first deal with $w(G)$.

TheOREm 4.1. $G(z)$ is spectraloid, i.e. $w(G)=r(G)$.

Proof. Let $\lambda \in W_{A}(G)$. Consider a corresponding sequence $v=\left(v_{\nu}\right) \in$ $H^{\mathbb{N}}, v \hat{\neq} 0$, with

$$
\lim _{\nu \rightarrow \infty} v_{\nu}^{*} G(\lambda) v_{\nu}=\lim _{\nu \rightarrow \infty} v_{\nu}^{*}\left[\lambda^{m} I-\sum_{j=0}^{m-1} C_{j} \lambda^{j}\right] v_{\nu}=0
$$

and

$$
v_{\nu}^{*} v_{\nu}=1, \quad \nu \in \mathbb{N} .
$$

Define $c_{j \nu}=v_{\nu}^{*} C_{j} v_{\nu}, j=0, \ldots, m-1$. The sequences

$$
\left(c_{j \nu}\right), \quad j=0, \ldots, m-1,
$$

are bounded. We can choose a suitable subsequence of $\left(v_{\nu}\right)$ such that the corresponding subsequences in (4.4) are convergent. Hence we may assume that the limits

$$
c_{j}^{(v)}=\lim _{\nu \rightarrow \infty} c_{j v_{\nu}}, \quad j=0, \ldots, m-1,
$$

exist. Define

$$
g^{(v)}(z)=z^{m}-\left(c_{m-1}^{(v)} z^{m-1}+\cdots+c_{1}^{(v)} z+c_{0}^{(v)}\right) .
$$

Then (4.2) is equivalent to $g^{(v)}(\lambda)=0$. Note that $g^{(v)}(z) \in \mathbb{R}[z]$ and

$$
c_{j}^{(v)} \geq 0, \quad j=0, \ldots, m-1 .
$$

Set $\rho=w(G)$. Assume $\sum_{j=0}^{m-1} C_{j} \neq 0$. Then $G(z) \neq I z^{m}$ and $\rho>0$. Because of (3.3) we have $\lambda \in W_{A}(G)$ for some $\lambda$ with $|\lambda|=\rho$. Let $v=\left(v_{\nu}\right) \in H^{\mathbb{N}}$, $v \neq 0$, be a corresponding sequence such that 4.2 holds, and let $g^{(v)}(z)$ be the polynomial in (4.6). Then $g^{(v)}(\lambda)=0$. Because of (4.7) there exists a unique positive root $\hat{\rho}$ of $g^{(v)}(z)$, and $r\left(g^{(v)}\right)=\hat{\rho}$ (see e.g. [38, p. 243], [37, p. 3]). Then $\hat{\rho}=\rho$. Otherwise we would have $w(G) \geq \hat{\rho}>\rho$. Hence

$$
g^{(v)}(\rho)=\lim _{\nu \rightarrow \infty} v_{\nu}^{*} G(\rho) v_{\nu}=0,
$$

and therefore $\rho \in W_{A}(G)$. Suppose $y^{*} G(\rho) y<0$ for some $y \neq 0$. If $u \in \mathbb{R}_{>}$ is sufficiently large then $y^{*} G(u) y>0$. Hence $y^{*} G(s) y=0$ for some $s>\rho$, and we would have $w(G)>\rho$. Therefore we obtain $G(\rho) \geq 0$. Hence (4.8) yields $\lim _{\nu \rightarrow \infty} G(\rho) v_{\nu}=0$. Thus $v=\left(v_{\nu}\right)$ is an approximate eigenvector 
of $G(z)$ corresponding to $\rho$. Hence $\rho \in \sigma_{A}(G)$. Therefore $\rho \leq r(G)$. Then $r(G) \leq w(G)$ implies $r(G)=w(G)$.

THEOREM 4.2. The numerical radius of $G(z)$ satisfies $w(G) \leq 1$ if and only if $G(1) \geq 0$, i.e.

$$
\sum_{j=0}^{m-1} C_{j} \leq I .
$$

Proof. Let $\rho=w(G)$. We know that $G(\rho) \geq 0$. Hence, if $0<\rho \leq 1$, then

$$
I \geq \sum_{j=0}^{m-1} C_{j} \rho^{j-m} \geq \sum_{j=0}^{m-1} C_{j},
$$

which proves (4.9). Now let $\lambda \in W_{A}(G)$ and let $v=\left(v_{\nu}\right) \in H^{\mathbb{N}}, v \hat{\neq} 0$, be a corresponding sequence such that 4.2 holds, and let $g^{(v)}(z)$ be the polynomial in 4.6). Then $g^{(v)}(\lambda)=0$. If 4.9 holds then

$$
\sum_{j=0}^{m-1} c_{j}^{(v)} \leq 1 .
$$

Hence Theorem 1.3 (i) yields $|\lambda| \leq 1$, and therefore $w(G) \leq 1$.

Corollary 4.3. We have $w(G)=1$ if and only if

$$
G(1) \geq 0 \text { and } \operatorname{Ker}_{A} G(1) \neq\{0\},
$$

or equivalently, if and only if

$$
\sum_{j=0}^{m-1} C_{j} \leq I \quad \text { and } \quad \operatorname{Ker}_{A}\left(I-\sum_{j=0}^{m-1} C_{j}\right) \neq\{0\} .
$$

Proof. We know from the proof of Theorem 4.1 that $w(G)=1$ implies 4.11. Conversely, if 4.11) holds, then $w(G) \leq 1$ (by Theorem 4.2), and $1 \in \sigma_{A}(G)$. Hence $w(G) \leq 1 \leq r(G)$ yields $w(G)=1$.

There is no loss of generality if we deal with operator polynomials $G(z)$ with $w(G)=1$. Let $0<w(G)=\rho$. Define $\tilde{G}(z)=\rho^{-m} G(\rho z)$. Then $\tilde{G}(z)=\rho^{-m} G(\rho z)$. Therefore $W(\tilde{G})=\rho^{-1} W(G)$ and $\sigma(\tilde{G})=\rho^{-1} \sigma(G)$, and $w(\tilde{G})=r(\tilde{G})=1$. If $\tilde{G}(z)=I z^{m}-\sum_{j=0}^{m-1} \tilde{C}_{j} z^{j}$, then $\tilde{C}_{j}=\rho^{-(m-j)} C_{j}$, $j=0, \ldots, m-1$. The coefficients of $\tilde{G}(z)$ have the following properties:

$$
\tilde{C}_{j} \geq 0, \quad j=0, \ldots, m-1, \quad \sum_{j=0}^{m-1} \tilde{C}_{j} \leq I .
$$

Corollary 4.4. We have $w(G)=\min \{s ; s \geq 0, G(s) \geq 0\}$. 
Proof. Set $q=\min \left\{s ; s \in \mathbb{R}_{\geq}, G(s) \geq 0\right\}$. Let $\rho=w(G)$. Then $G(\rho) \geq 0$. Hence $q \leq \rho$. Suppose $G(s) \geq 0$. Then Theorem 4.2 implies $\rho \leq s$, and we obtain $q \geq \rho$. Hence $q=\rho$. Note that $G(s) \geq 0$ for all $s \geq \rho$.

Corollary 4.5. If $\sum_{j=0}^{m-1} C_{j} \ll I$ then $w(G)<1$.

Proof. The assumption implies that the inequality in 4.10 is strict. Thus $g^{(v)}(1)=\sum_{j=0}^{m-1} c_{j}^{(v)}<1$. Therefore $|\lambda|<1$ for all $\lambda \in W_{A}(G)$. Since $W_{A}(G)$ is closed we obtain $w(G)<1$.

In general, the inequality $\sum_{j=0}^{m-1} C_{j}<I$ is not sufficient for $w(G)<1$.

EXAMPLE 4.6. Let $H=\ell_{2}$ and

$$
C_{0}=\operatorname{diag}(1 / 2,2 / 3,3 / 4,4 / 5, \ldots) .
$$

Then $0<C_{0}<I$, and the inequality $C_{0} \ll I$ is not satisfied. Consider $G(z)=z I-C_{0}$. Then

$$
I-C_{0}=\operatorname{diag}(1 / 2,1 / 3, \ldots, 1 / k, \ldots)>0
$$

implies $w(G) \leq 1$. We have noted in Example 3.1 that $G(1) e=\left(I-C_{0}\right) e \hat{=} 0$. Hence $1 \in \sigma_{A}(G)$, and $w(G)=1$.

4.2. The spectrum on the unit circle. In this section we consider operator polynomials with $r(G)=w(G)=1$. Thus we assume

$$
C_{j} \geq 0, \quad j=0, \ldots, m-1, \quad \sum_{j=0}^{m-1} C_{j} \leq I,
$$

and

$$
\operatorname{Ker}_{A}\left(I-\sum_{j=0}^{m-1} C_{j}\right) \neq\{0\} .
$$

Hence $\sigma(G) \cap \partial \mathbb{D} \neq \emptyset$. Let $v=\left(v_{\nu}\right) \in H^{\mathbb{N}}, v \hat{\neq} 0$, be given. We define

$$
M(v)=\{\mu ; \mu \in \partial \mathbb{D}, G(\mu) v \stackrel{=}{=}\} .
$$

Then $M(v)$ consists of those approximate characteristic values $\mu$ of $G(z)$ which lie on the unit circle and have $v$ as a corresponding approximate eigenvector. In the proof of Theorem 4.1 we have seen that $w(G)=\rho=1$, and $|\lambda|=1$ and $G(\lambda) v \hat{=} 0$ imply $G(1) v \hat{=} 0$. Hence we have $M(v) \neq \emptyset$ if and only if

$$
G(1) v=\left(I-\sum_{j=0}^{m-1} C_{j}\right) v \hat{=} 0, \quad v \hat{\neq} 0 .
$$

We may assume that $v$ is a sequence satisfying (4.3) and that the limits 4.5 . exist. If $\lambda \in \sigma_{A}(G)$ and $v$ is a corresponding approximate eigenvector then 
$G(\lambda) v \hat{=} 0$ implies $C_{j} v \hat{\neq} 0$ for some $j, 0 \leq j \leq m-1$. Let $t_{v}$ be defined by

$$
C_{0} v \hat{=} \cdots \hat{=} C_{t_{v}-1} v \hat{=} 0, \text { and } C_{t_{v}} v \hat{\neq} 0
$$

Note that $C_{0} \gg 0$ implies $t_{v}=0$. We now describe the structure of $M(v)$ and generalize Theorem 1.3 (iv).

Theorem 4.7. Assume 4.15). Set

$$
d_{v}=\operatorname{gcd}\left(\left\{j ; C_{t_{v}+j} v \hat{\neq} 0, j=0, \ldots, m-t_{v}-1\right\} \cup\left\{m-t_{v}\right\}\right) .
$$

Then $M(v)=E_{d_{v}}$.

Proof. Let $g^{(v)}(z)=z^{m}-\sum_{j=0}^{m-1} c_{j}^{(v)} z^{j}$ be the polynomial in (4.6). Then $\lambda \in M(v)$ implies $g^{(v)}(\lambda)=0$. From 4.16$)$ it follows that $c_{t}^{(v)}>0, c_{j}^{(v)}=0$, if $j<t$. We apply Theorem 1.3(iv) to determine the unimodular roots of $g^{(v)}(z)$. Set

$$
\hat{d}_{v}=\operatorname{gcd}\left(\left\{j ; c_{t_{v}+j}^{(v)} \neq 0, j=0, \ldots, m-t_{v}-1\right\} \cup\left\{m-t_{v}\right\}\right) .
$$

Then (1.6) yields $E_{\hat{d}_{v}}=\left\{\lambda ; g^{(v)}(\lambda)=0,|\lambda|=1\right\}$. Because of $C_{j} \geq 0$ we have $c_{j}^{(v)}=\lim _{\nu \rightarrow \infty} v_{\nu}^{*} C_{j} v_{\nu}=0$ if and only if $\lim _{\nu \rightarrow \infty} C_{j} v_{\nu}=0$. Hence $\hat{d}_{v}=d_{v}$, and therefore $M(v) \subseteq E_{d_{v}}$.

To prove the inclusion $E_{d_{v}} \subseteq M(v)$, we first note that 4.16) and 4.17) imply

$$
G(z) v \hat{=} z^{t_{v}}\left[z^{\ell d_{v}} I-\sum_{j=0}^{\ell-1} C_{j d_{v}} z^{j d_{v}}\right] v .
$$

If $\lambda \in E_{d_{v}}$ then $\lambda^{d_{v}}=1$, and therefore 4.18 yields $G(\lambda) v=G(1) v$. Then 4.15 implies $G(\lambda) v \stackrel{\wedge}{=}$. Hence $\lambda \in M(v)$.

The assumption $r(G)=w(G)=1$ implies that approximate characteristic values of $G(z)$ on the unit circle are on the boundary of the numerical range of $G(z)$. Therefore we can take advantage of results of Section 3. An immediate consequence of Theorem 3.7(i) is the following.

Theorem 4.8. If $\lambda \in \sigma(G)$ and $|\lambda|=1$, then $\lambda \notin \sigma_{R}(G)$, i.e.

$$
\sigma_{R}(G) \cap \partial \mathbb{D}=\emptyset .
$$

Thus, if the spectrum of $G(z)$ on the unit circle is nonempty then its elements are approximate characteristic values. The next theorem shows that all of them are approximately normal and semisimple.

Theorem 4.9. If $\lambda \in \sigma(G)$ and $|\lambda|=1$, then

$$
\operatorname{Ker}_{A} G(\lambda)=\operatorname{Ker}_{A} G(\lambda)^{*} \quad \text { and } \quad \operatorname{Ker} G(\lambda)=\operatorname{Ker} G(\lambda)^{*},
$$

and $\lambda$ is approximately semisimple. 
Proof. The identities 4.19) are taken from Theorem 3.7(ii). We apply Proposition 2.5 to show that $\lambda$ is approximately semisimple. Assume that $v=\left(v_{\nu}\right)$ is such that 4.3 holds and that the limits $c_{j}^{(v)}$ in 4.5 exist. Let $g^{(v)}(z)$ be the corresponding polynomial 4.6 . It follows from Theorem 1.3 (iii) that $\lambda$ is a simple root of $g^{(v)}(z)$. Hence $\left(g^{(v)}\right)^{\prime}(\lambda) \neq 0$. Therefore $\lim _{\nu \rightarrow \infty} v_{\nu}^{*} G^{\prime}(\lambda) v_{\nu} \neq 0$, which amounts to condition 2.14.

We record two observations, which will be used later.

LEMMA 4.10.

(i) If $C_{0} \gg 0$ then $\sigma(G) \cap \partial \mathbb{D} \subseteq E_{m}$, where $m=\operatorname{deg} G$.

(ii) If $C_{0} \gg 0$ and $C_{1} \gg 0$ then $\sigma(G) \cap \partial \mathbb{D} \subseteq\{1\}$.

Proof. Suppose $\sigma(G) \cap \partial \mathbb{D} \neq \emptyset$, that is, $1 \in \sigma_{A}(G)$. Let $v$ be an approximate eigenvector corresponding to 1 . Then $C_{0} \gg 0$ implies $t_{v}=0$. Hence $d_{v} \mid m$, and therefore $M(v) \subseteq E_{d_{v}} \subseteq E_{m}$. If $C_{j} \gg 0, j=0,1$, then $d_{v}=1$. Hence $M(v)=\{1\}$.

In Example 2.4 we considered a polynomial $G(z)=z^{2} I-\left(z C_{1}+C_{0}\right)$ and extracted a factor $z+1$ from $G(z) e \in H[z]$. A general factorization result is given in 4.20 below. It extends the identity 1.7 in Theorem 1.3(iv).

Theorem 4.11. Suppose $G(1) v \hat{=} 0, v \hat{\neq} 0$. Let $t_{v}$ and $d_{v}$ be defined by (4.16) and (4.17), respectively. If $m-t_{v}=\ell d_{v}$ then

$$
G(z) v \hat{=} z^{t_{v}}\left(z^{d_{v}}-1\right) p\left(z^{d_{v}}\right),
$$

where $p(z)=\left(p_{\nu}(z)\right)$ is a sequence in $H[z]$ and

$$
p\left(\lambda^{d_{v}}\right) \hat{\neq} 0 \quad \text { if } \quad|\lambda|=1 .
$$

Proof. In 4.18 we have seen that $G(z) v \hat{=} z^{t_{v}}\left[I z^{\ell d_{v}}-\sum_{j=0}^{\ell-1} C_{j d_{v}} z^{j d_{v}}\right] v$. Hence $G(z) v \hat{=} z^{t_{v}} q\left(z^{d_{v}}\right)$ for some sequence $q(z)=\left(q_{\nu}(z)\right)$ in $H[z]$. If $\lambda^{d_{v}}-1=0$ then $G(\lambda) v \hat{=} 0$, and we obtain $q(z)=\left(z^{d_{v}}-1\right) p(z)$. It remains to show that the sequence $p\left(z^{d_{v}}\right)$ in 4.20 satisfies (4.21). Suppose $p\left(\lambda^{d_{v}}\right) \hat{=} 0$ for some $\lambda \in \partial \mathbb{D}$. Then $\lambda \in M(v)$ and therefore $\lambda \in E_{d_{v}}$, i.e. $\lambda^{d_{v}}-1=0$. Hence $G(\lambda) v \hat{=} G^{\prime}(\lambda) v \hat{=} 0$. Then $(v, v)$ would be an approximate Jordan chain of length 2 corresponding to $\lambda$. Hence $\lambda \in \sigma_{A}(G) \cap \partial \mathbb{D}$ would not be approximately semisimple, in contradiction to Theorem 4.9 .

\subsection{An operator polynomial with nonempty residual spectrum.}

We have seen in Theorem 4.8 that the residual spectrum of $G(z)$ on the unit circle is empty. In this section we construct an operator polynomial of the form 4.1 with the properties 4.13) and 4.14, which has a residual spectrum (contained in the open unit disk).

EXAMPLE 4.12. Let $H=\ell_{2}$. We construct a monic operator polynomial

$$
G(z)=I z^{3}-\left(C_{2} z^{2}+C_{1} z+C_{0}\right) \in \mathcal{L}(H)[z]
$$


with selfadjoint positive semidefinite coefficients $C_{j}$ satisfying

$$
C_{2}+C_{1}+C_{0}=I
$$

such that

$$
\sigma_{R}(G) \cap \mathbb{D} \neq \emptyset,
$$

i.e. such that there exists a $\lambda \in \mathbb{D}$ with $0 \in \sigma_{R}(G(\lambda))$. Let

$$
S_{+}:\left(z_{1}, z_{2}, z_{3}, \ldots\right) \mapsto\left(0, z_{1}, z_{2}, \ldots\right)
$$

be the right shift and

$$
S_{-}:\left(z_{1}, z_{2}, z_{3}, \ldots\right) \mapsto\left(z_{2}, z_{3}, z_{4}, \ldots\right)
$$

the left shift on $\ell_{2}$. It is known (see [34, p. 420]) that $\mathbb{D} \subseteq \sigma_{R}\left(S_{+}\right)$. In particular, $0 \in \sigma_{R}\left(S_{+}\right)$. This can be seen as follows. The map $S_{+}: \ell_{2} \rightarrow \ell_{2}$ is injective, and range $\left(S_{+}\right)=\left\langle e_{1}\right\rangle^{\perp}$ is not dense in $\ell_{2}$. Set

$$
U=\frac{1}{2}\left(S_{+}+S_{-}\right) \quad \text { and } \quad V=\frac{1}{2}\left(S_{+}-S_{-}\right) .
$$

Then $S_{+}^{*}=S_{-}$implies $U^{*}=U$ and $(i V)^{*}=i V$. Clearly, $U+V=S_{+}$. Let $0<\alpha<\frac{1}{2}$. Define $d(z)=z^{3}-\left[(1-2 \alpha) z^{2}+\left(2 \alpha-2 \alpha^{2}\right) z+2 \alpha^{2}\right]$ and

$$
D(z)=I z^{3}-\left(D_{2} z^{2}+D_{1} z+D_{0}\right)=d(z) I .
$$

Then $\lambda=-(1+i) \alpha$ is a root of $d(z)$, and

$$
d(z)=(z-\lambda)(z-\bar{\lambda})(z-1)=\left(z^{2}+2 \alpha z+2 \alpha^{2}\right)(z-1) .
$$

From $D(1)=0$ it follows that

$$
D_{2}+D_{1}+D_{0}=I .
$$

Set $\kappa=1+2 \alpha+2 \alpha^{2}$. The polynomials

$$
p(z)=z^{2}+2 \alpha z-(1+2 \alpha) \quad \text { and } \quad q(z)=\frac{1+\alpha}{\alpha} z^{2}-\frac{1}{\alpha} z-1
$$

satisfy

$$
p(1)=q(1)=0, \quad p(\lambda)=-\kappa, \quad q(\lambda)=i \kappa .
$$

Define

$$
E(z)=E_{2} z^{2}+E_{1} z+E_{0}=p(z) U+q(z) i V
$$

such that

$$
\begin{aligned}
& E_{0}=-(1+2 \alpha) U-\quad i V, \\
& E_{1}=2 \alpha U-\frac{1}{\alpha} i V, \\
& E_{2}=\quad U \quad+\left(\frac{1}{\alpha}+1\right) i V .
\end{aligned}
$$

Then 4.25) implies

$$
E_{2}+E_{1}+E_{0}=0
$$


and

$$
E(\lambda)=p(\lambda) U+q(\lambda) i V=-\kappa U+i \kappa i V=-\kappa S_{+} .
$$

We consider the operator polynomial

$$
G(z)=D(z)-\epsilon E(z), \quad \epsilon>0 .
$$

The coefficients of $G(z)$ have the form $C_{j}=D_{j}-\epsilon E_{j}, j=0,1,2$. Because $0<\alpha<1 / 2$ the operators

$$
D_{0}=2 \alpha^{2} I, \quad D_{1}=2 \alpha(1-\alpha) I, \quad D_{2}=(1-2 \alpha) I
$$

are strictly positive definite. The operators $E_{j}$ are self-adjoint. We assume that $\epsilon>0$ is sufficiently small such that $C_{j} \gg 0, j=0,1,2$. From (4.24) and 4.26 we obtain 4.22). To prove 4.23 we evaluate $G(z)$ at the point $\lambda$. From $D(\lambda)=0$ and (4.27) we deduce that $G(\lambda)=\epsilon \kappa S_{+}$, and consequently $0 \in \sigma_{R}(G(\lambda))$.

Let us determine the spectrum of $G(z)$ on the unit circle. From 4.22 it follows that $1 \in \sigma(G)$. Because of $C_{0} \gg 0$ and $C_{1} \gg 0$ we can apply Lemma 4.10, and we see that 1 is the only element of $\sigma(G)$ that lies on the unit circle.

5. The Eneström-Kakeya theorem in Hilbert space. Theorems 1.1 and 1.2 have been extended to matrix polynomials ([14], 42]). In this section we obtain more general results for operator polynomials.

THEOREM 5.1. Let

$$
H(z)=A_{m-1} z^{m-1}+\cdots+A_{1} z+A_{0}
$$

be an operator polynomial with selfadjoint coefficients $A_{j} \in \mathcal{L}(H)$. Assume

$$
A_{m-1} \gg 0, \quad A_{m-1} \geq A_{m-2} \geq \cdots \geq A_{0} \geq 0 .
$$

Then:

(i) $r(H) \leq 1$ and $1 \notin \sigma(H)$.

(ii) The residual spectrum of $H(z)$ on the unit circle is empty.

(iii) If $\lambda \in \sigma(H)$ and $|\lambda|=1$, then $\operatorname{Ker}_{A} H(\lambda)=\operatorname{Ker}_{A} H(\lambda)^{*}$ and $\lambda$ is approximately semisimple.

(iv) Suppose $A_{0} \gg 0$. Then $\lambda \in \sigma(H)$ and $|\lambda|=1$ imply $\lambda^{m}=1$.

Proof. (i) From $A_{m-1} \gg 0$ it follows that $A_{m-1}^{1 / 2} \gg 0$, and therefore $R:=A_{m-1}^{-1 / 2} \in \mathcal{L}(H), R=R^{*}$. Set $\tilde{A}_{j}=R^{*} A_{j} R, j=0, \ldots, m-1$. Then

$$
R^{*} H(z) R=I z^{m-1}+\sum_{j=0}^{m-2} \tilde{A}_{j} z^{j} .
$$

Thus, it suffices to consider (5.1) with $A_{m-1}=I$ and

$$
I \geq A_{m-2} \geq \cdots \geq A_{0} \geq 0 \text {. }
$$


As in the case of the polynomial $h(z)$ in $(1.1)$ one can use the multiplier $z-1$. Define $G(z)=(z-1) H(z)$. Then $G(z)=I z^{m}-\sum_{j=0}^{m-1} C_{j} z^{j}$, and $C_{0}=A_{0}$, and $C_{j}=A_{j}-A_{j-1} \geq 0, j=1, \ldots, m-1$, and $C_{0}+\cdots+C_{m-1}=I$, and $1 \in \sigma_{P}(G)$. Moreover,

$$
\sigma(G)=\sigma(H) \cup\{1\} .
$$

From Corollary 4.3 we get $r(G)=w(G)=1$. Hence (5.3) implies $r(H) \leq 1$. Let us show that $1 \notin \sigma(H)$. Because of 5.2 we have $H(1)=\sum_{j=0}^{m-1} A_{j} \gg 0$. Hence $H(1)$ has a bounded inverse and therefore $0 \notin \sigma(H(1))$.

It is obvious that (ii) follows from (4.8). For (iii) we refer to Theorem 4.9 , and (iv) is a consequence of Lemma 4.10 (i).

We now extend Theorem 1.2 to a result on operator polynomials. We focus on an approximate eigenvector $v$ of $H(z)$. With regard to 1.3 we make the assumptions

$$
\begin{aligned}
& A_{0} v \hat{=} \cdots \hat{=} A_{r_{1}-1} v, A_{r_{1}-1} v \hat{\neq} A_{r_{1}} v \\
& A_{r_{1}} v \hat{=} \cdots \hat{=} A_{r_{2}-1} v, A_{r_{2}-1} v \hat{\neq} A_{r_{2}} v, \ldots, \\
& \quad A_{r_{s}-1} v \hat{\neq} A_{r_{s}} v, A_{r_{s}} v \hat{=} \cdots \hat{=} A_{m-1} v .
\end{aligned}
$$

THEOREM 5.2. Suppose the coefficients of $H(z)$ satisfy

$$
A_{m-1} \gg 0 \text { and } A_{m-1} \geq A_{m-2} \geq \cdots \geq A_{0} \gg 0 .
$$

Let $\lambda \in \sigma(H)$ and $|\lambda|=1$, and let $v$ be a corresponding approximate eigenvector. Let $r_{1}, \ldots, r_{s}$ be given by (5.4). Define $k=\operatorname{gcd}\left(r_{1}, \ldots, r_{s}, m\right)$. Then $\lambda^{k}=1$, and

$$
H(z) v \hat{=}\left(1+z+\cdots+z^{k-1}\right) p\left(z^{k}\right),
$$

where $p(z)=\left(p_{\nu}(z)\right) \subseteq H[z]$ is a sequence with

$$
\lim _{\nu \rightarrow \infty} p_{\nu}\left(\lambda^{k}\right) \neq 0 \quad \text { if }|\lambda|=1 .
$$

Proof. Again, we can assume $A_{m-1}=I$, and pass from $H(z)$ to $G(z)$. The coefficients of $G(z)$ satisfy (5.2). Therefore (5.4) is equivalent to

$$
\begin{aligned}
\left\{1, \ldots, r_{1}-1, r_{1}+1, \ldots, r_{2}-1, \ldots,\right. & \left.r_{s}+1 \ldots, m-1\right\} \\
& =\left\{j ; 0 \leq j \leq m-1, C_{j} v \hat{=} 0\right\},
\end{aligned}
$$

and we have $\left\{j ; 0 \leq j \leq m-1, C_{j} v \hat{\neq} 0\right\}=\left\{r_{1}, \ldots, r_{s}\right\}$. Hence

$$
k=\operatorname{gcd}\left(\left\{j ; 0 \leq j \leq m-1, C_{j} v \hat{\neq} 0\right\} \cup\{m\}\right) .
$$

Then Theorem 4.11 and $t_{v}=0$ yield

$$
G(z) v \hat{=}(z-1) H(z) v \hat{=}\left(z^{k}-1\right) p\left(z^{k}\right) \hat{=}(z-1)\left(1+z+\cdots+z^{k-1}\right) p\left(z^{k}\right) .
$$

Finally, 4.21 implies that the sequence $p\left(z^{k}\right)=\left(p_{\nu}\left(z^{k}\right)\right)$ satisfies (5.5). 
Acknowledgments. We thank G. Dirr for valuable comments.

\section{References}

[1] N. Anderson, E. B. Saff, and R. S. Varga, On the Eneström-Kakeya theorem and its sharpness, Linear Algebra Appl. 28 (1979), 5-16.

[2] - , 一, 一, An extension of the Eneström-Kakeya theorem and its sharpness, SIAM J. Math. Anal. 12 (1981), 10-22.

[3] G. Bachman and L. Narici, Functional Analysis, Academic Press, New York, 1966.

[4] H. Baumgärtel, Analytic Perturbation Theory for Matrices and Operators, Operator Theory Adv. Appl. 15, Birkhäuser, Basel, 1985.

[5] S. K. Berberian, Lectures in Functional Analysis and Operator Theory, Springer, New York, 1974.

[6] —, Approximate proper vectors, Proc. Amer. Math. Soc. 13 (1962), 111-114.

[7] S. K. Berberian and G. H. Orland, On the closure of the numerical range of an operator, Proc. Amer. Math. Soc. 18 (1967), 499-503.

[8] R. Bhatia, Matrix Analysis, Springer, New York, 1997.

[9] P. Borwein and T. Erdélyi, Polynomials and Polynomial Inequalities, Springer, New York, 1995.

[10] M. Brambilla, A. Giovannini, and R. Ugoccioni, Maps of zeros of the grand canonical partition function in a statistical model of high energy collisions, J. Phys. G 32 (2006), 859-870.

[11] A. J. Carpenter, R. S. Varga, and J. Waldvogel, Asymptotics for the zeros of the partial sums of $e^{z}$, Rocky Mountain J. Math. 21 (1991), 99-120.

[12] F. Chatelin, Spectral Approximation of Linear Operators, Academic Press, New York, 1983.

[13] K. Chinen, An abundance of invariant polynomials satisfying the Riemann hypothesis, Discrete Math. 308 (2008), 6426-6440.

[14] G. Dirr and H. K. Wimmer, An Eneström-Kakeya theorem for hermitian polynomial matrices, IEEE Trans. Automat. Control 52 (2007), 2151-2153.

[15] K.-J. Engel and R. Nagel, A Short Course on Operator Semigroups, Springer, New York, 2006.

[16] M. Enomoto, M. Fujii, and K. Tamaki, On normal approximate spectrum, Proc. Japan Acad. 48 (1972), 211-215.

[17] F. O. Farid, On the numerical range of operator polynomials, Linear Multilinear Algebra 50 (2002), 223-239.

[18] M. Fujii and F. Kubo, Operator norms as bounds for roots of algebraic equations, Proc. Japan Acad. 49 (1973), 805-808.

[19] M. Fujii and K. Tamaki, On normal approximate spectrum, III, ibid. 48 (1972), 389-393.

[20] T. Furuta, Invitation to Linear Operators, Taylor \& Francis, New York, 2001.

[21] T. Furuta and M. Nakamura, An operator version of the Eneström-Kakeya theorem, Math. Japon. 37 (1992), 459-497.

[22] K. E. Gustafson and D. K. M. Rao, Numerical Range, Springer, New York, 1996.

[23] P. R. Halmos, A Hilbert Space Problem Book, Van Nostrand, Princeton, NJ, 1967.

[24] P. D. Hislop and I. M. Sigal, Introduction to Spectral Theory. With Applications to Schrödinger Operators, Springer, New York, 1996.

[25] B. Huppert, Angewandte Lineare Algebra, de Gruyter, Berlin, 1990. 
[26] A. Hurwitz, Über einen Satz des Herrn Kakeya, Tôhoku Math. J. 4 (1913), 89-93; reprinted in: Mathematische Werke von A. Hurwitz, 2. Band, Birkhäuser, Basel, 1933, 627-631.

[27] V. Kozlov and V. Maz'ya, Differential Equations with Operator Coefficients, Springer, Berlin, 1999.

[28] U. Krause, A local-global stability principle for discrete systems and difference equations, in: B. Aulbach, S. Elaydi, and G. Ladas (eds.), Proc. Sixth Internat. Conf. on Difference Equations (Augsburg, 2001), CRC Press, Boca Raton, FL, 2004, 167-180.

[29] C.-K. Li and L. Rodman, Numerical range of matrix polynomials, SIAM J. Matrix Anal. Appl. 15 (1994), 1256-1265.

[30] W. A. Majewski, Quantum dynamical maps and return to equilibrium, Acta Phys. Polon. B 32 (2001), 1467-1474.

[31] M. Marden, Geometry of Polynomials, 2nd ed., Math. Surveys 3, Amer. Math. Soc., Providence, RI, 1966.

[32] J. Maroulas and P. Psarrakos, The boundary of the numerical range of matrix polynomials, Linear Algebra Appl. 267 (1997), 101-111.

[33] H. Nakazato and P. Psarrakos, On the shape of numerical range of matrix polynomials, ibid. 338 (2001), 105-123.

[34] A. W. Naylor and G. R. Sell, Linear Operator Theory in Engineering and Science, Springer, New York, 1982.

[35] A. M. Ostrowski, Solutions of Equations in Euclidean and Banach Spaces, Academic Press, New York, 1973.

[36] S.-C. Pei and P.-H. Wang, Closed-form design of allpass fractional delay filters, IEEE Signal Process. Lett. 11 (2004), 788-791.

[37] V. V. Prasolov, Polynomials, Springer, New York, 2004.

[38] Q. I. Rahman and G. Schmeisser, Analytic Theory of Polynomials, Oxford Univ. Press, Oxford, 2002.

[39] M. Reed and B. Simon, Methods of Modern Mathematical Physics, I: Functional Analysis, Academic Press, New York, 1972.

[40] W. Rudin, Functional Analysis, McGraw-Hill, New York, 1973.

[41] S. Wada, An alternative proof of Kakeya's theorem and the Lohmann-Ruchti effect, Bull. Osaka Prefect. Univ. Ser. D 13 (1969), 1-5.

[42] H. K. Wimmer, Discrete-time stability of a class of hermitian polynomial matrices with positive semidefinite coefficients, in: V. Olshevsky and E. Tyrtyshnikov (eds.), Matrix Methods: Theory, Algorithms and Applications, World Sci., Singapore, 2010, 409-414.

[43] K. Zhu, An Introduction to Operator Algebras, CRC Press, Boca Raton, FL, 1993.

Jan Swoboda

Max-Planck-Institut für Mathematik

D-53111 Bonn, Germany

E-mail: swoboda@mpim-bonn.mpg.de
Harald K. Wimmer

Mathematisches Institut Universität Würzburg

D-97074 Würzburg, Germany

E-mail: wimmer@mathematik.uni-wuerzburg.de

Received September 24, 2009

Revised version January 13, 2010 